\title{
Exploring Algorithm Comprehension: Linking Proof and Program Code
}

\author{
Philipp Kather \\ philipp.kather@uni-muenster.de \\ Westfälische Wilhelms-Universität \\ Münster, Germany
}

\author{
Jan Vahrenhold \\ jan.vahrenhold@uni-muenster.de \\ Westfälische Wilhelms-Universität \\ Münster, Germany
}

\begin{abstract}
An algorithm consists of the description of a process solving a welldefined problem and a chain of reasoning regarding one or more properties of this process, such as efficiency or correctness. Often, this chain of reasoning is presented as a formal proof. Understanding the links between this reasoning and the process is a critical task for understanding algorithm comprehension. In this article, we present results from a qualitative study in which we explored how and where links between parts of the process and parts of the chain of reasoning are established by a reader attempting to comprehend an algorithm. We relate our findings to research in program and proof comprehension and observe that the connection between process and reasoning introduces another dimension of comprehension not covered by previous work. We identify potential mechanisms in this comprehension process and derive suggestions for how to support comprehension when teaching algorithms.
\end{abstract}

\section{CCS CONCEPTS}

- Social and professional topics $\rightarrow$ Computing education

\section{KEYWORDS}

Algorithm comprehension, program comprehension, proof comprehension.

\section{ACM Reference Format:}

Philipp Kather and Jan Vahrenhold. 2021. Exploring Algorithm Comprehension: Linking Proof and Program Code. In 21st Koli Calling International Conference on Computing Education Research (Koli Calling '21), November 18-21, 2021, Joensuu, Finland. ACM, New York, NY, USA, 10 pages. https://doi.org/10.1145/3488042.3488061

\section{INTRODUCTION}

Algorithms are ubiquitous in Computer Science. Yet, the degree of formality with which they are introduced and analyzed varies between courses, ranging from intuitive descriptions to formal verification. In this paper, we focus on the more formal perspective usually taken in advanced courses on algorithms in which algorithms are analyzed with respect to, e. g., time or space complexity, correctness, or optimality of the result produced. Since such courses often address algorithms on different levels of abstraction, including

Permission to make digital or hard copies of part or all of this work for personal or classroom use is granted without fee provided that copies are not made or distributed for profit or commercial advantage and that copies bear this notice and the full citation on the first page. Copyrights for third-party components of this work must be honored. For all other uses, contact the owner/author(s).

Koli Calling '21, November 18-21, 2021, foensuu, Finland

(c) 2021 Copyright held by the owner/author(s).

ACM ISBN 978-1-4503-8488-9/21/11.

https://doi.org/10.1145/3488042.3488061 prose descriptions, pseudo-code, program code, and mathematical notations, learners anecdotally perceive these courses as rather challenging. From an instructor's perspective, it thus would be desirable to understand how learners comprehend algorithms because instruction can then be aligned accordingly.

To describe what we consider to be an algorithm and to distinguish an "algorithm" from a "program" commonly addressed in courses for novice programmers and studied in the program comprehension literature, we use the following definition [20]:

Algorithm. An algorithm consists of a process solving a welldefined problem along with a chain of reasoning regarding one or more properties of this process, such as efficiency or correctness. The process may be represented verbatim or as succinct (pseudo-)code, possibly augmented by comments and/or accompanied by a prose description. The chain of reasoning may be represented as a formal proof or a proof sketch. To make such a proof worthwhile, it should be nontrivial for the intended reader of the algorithm.

In a previous grounded-theory-based study, we found that comprehension of algorithms is based on linking the proof and the process [20]. In the present paper, we further explore this connection and relate it to research on program comprehension and proof comprehension. Moreover, we explore how guiding principles known from multimedia comprehension [28] can be used to also guide algorithm comprehension. Barring the discussion in Section 2, only little work has been done to understand the comprehension of an algorithm in the sense of the above definition. We thus conducted an exploratory qualitative study of advanced students attempting to comprehend algorithms and focused on the guiding research question: Which elements of the proof are connected to which elements of the program when reading an algorithm?

\section{RELATED WORK}

Historically, programs have been referred to as "algorithms", even when they are presented without an accompanying proof; a prominent example is the "long division algorithm" in elementary mathematics which is usually reduced to its process. As a consequence, there is some body of research on "algorithm comprehension" that actually is research on "program comprehension". For example, Narayanan and Hegarty studied how to support learners' comprehension of program code snippets describing the procedure of well-known algorithms, e.g., Quicksort, by providing simulations on various levels of abstraction [28, pp. 298-307]. As they did not present or discuss any chain of reasoning related to the process, their research classifies as research on "program comprehension". 
Research on algorithm comprehension. Fant [10] notes that the term algorithm has been used with various meanings in the Computer Science (Education) literature. We thus carefully reviewed the literature and found that research on "algorithms" in the sense of our working defintion is rare. Haberman et al. extended the process-object regarding the concept of algebraic functions [35] to describe the concept of algorithms: An algorithm can be viewed as an operational process entity (embodying a 'how' view), as well as an object entity that embodies an I/O relationship (a 'what' view) [17, p. 78]. However, learners' concepts of algorithms were found to differ. They did not consider algorithms based on mathematical properties to qualify as a proper algorithm, because it does not emulate a process [17, p. 77]. Yet, Ginat and Blau showed that such mathematical properties support developing "efficient (and often more elegant) algorithms" [15, pp. 237-238]. Haberman et al. thus concluded that, "in order to construct a broad comprehensive view of the notion of an algorithm, one should adopt the perspective that the solution of an algorithmic problem consists of both: (a) a well-designed algorithm, and (b) an accompanying written document especially reflects problem analysis and solution characteristics - in particular, correctness, clarity, and efficiency." [17, p. 74]. Our above definition of the term "algorithm" resembles this perspective.

Perrenet et al. defined levels of abstraction based on the processobject duality and found more advanced students to have a more abstract perspective [30, pp. 64-65]. Their results imply that a study aiming at understanding on different levels of abstraction should include advanced students as participants (see Section 3).

Research related to algorithms mainly focused on visualization of the process or supporting the comprehension of complexity (see $[5,16,36]$ and the references therein). Whereas visualizationbased research focuses on the process alone, research on complexity includes the process-object duality by considering a property of the algorithm. To the best of our knowledge, however, no research was conducted studying the connection between the accompanying document, i. e., the proof sketch, and the process.

Another related line of research stems from applying learning theories to algorithm comprehension. Processes described by $\mathrm{Pi}-$ aget [31] were identified in algorithm comprehension [8, 9]. Concepts from multimedia learning, e. g., simulations, were found to support understanding the process underlying an algorithm [28].

As findings from related fields can increase sensitivity in qualitative work, we also discuss two obviously related fields: program comprehension and proof comprehension. We point out, however, that our previous work [20] strongly suggests that algorithm comprehension cannot be explained solely by one of these.

Research on program comprehension. Since the process description of an algorithm can be represented as a program, findings from program comprehension are likely to be relevant to algorithm comprehension. Program comprehension research goes back into the 1980s-see, e. g., [23, 29, 38]. Some articles use the term "algorithm" to refer to the procedure underlying the algorithm (e.g. [7, 28]). Therefore, we consider those articles to be related to program comprehension, i. e., to attach meaning to a piece of program code. Other articles focus on the comprehension of longer pieces of program code $[38,39]$ or less complex tasks such as maintaining or modifying data-see, e. g., [3, 23, 29]. In none of these studies, any proof or reasoning was provided and only "little documentation was included" [3, p. 127]. This imposes limitations regarding the extent to which findings program comprehension can be generalized.

"Levels of abstraction" are a recurring theme in program comprehension: The comprehension process can be described as bottomup from details to higher levels of abstractions (see, e. g., [3, 29]) or hypothesis-driven and top-down [2]. Letovsky described these strategies as means to connect layers of abstraction "that show how the goals are decomposed into subgoals and ordered together" [23, p. 332]. This notion of abstraction allowed Schulte et al. to relate those and other theories on program comprehension to the block model [33]. In this model, comprehension is characterized in terms of abstraction, ranging from understanding details such single operations over understanding blocks of program code in isolation to "understanding the overall structure of the program" [32, p. 150]. As abstraction is central to theories on program comprehension, we were sensitive to this concept throughout our exploratory study.

Research on proof comprehension. Models on proof comprehension characterize comprehension as knowing "how a proof operates and why a proof is right, in addition to knowing what a proof can prove" [40, p.60]. Based on literature reviews in mathematics education and reading comprehension as well as based on interviews with mathematicians, Yang and Lin developed a theory of geometry proof comprehension which was operationalized and validated for academically much younger subjects in high school [40]. Mejía-Ramos et al. [26] extended this theory to more advanced mathematics and created an instrument to assess comprehension of a proof of a theorem in elementary number theory. Both theories illustrate comprehension as building a mental representation of several levels. The lower levels are concerned with comprehending symbols and properties, followed by classifying them as premises, applied properties and conclusion; the third level is reached once the logical relation of these is understood and critical ideas are summarized [40]. Mejía-Ramos et al. [26] extend this level and assess the ability to create appropriate summaries of a proof, to divide a proof into meaningful sections, and to transfer a proof.

Implications. Both program and proof comprehension describe comprehension based on levels of abstraction and thus one should consider levels of abstraction when studying algorithm comprehension. One should be aware, though, that proofs of algorithms are concerned with a dynamic entity-the program-whereas the mathematical proofs in the studies described above were concerned with static entities. It thus remains open to which results from proof comprehension carry over to algorithm comprehension. Similarly, we illustrated that algorithm comprehension cannot be explained based on program comprehension only [20]: The interaction between proof and program appears to be one of the main reasons for this. We thus ask: Which elements of the proof are connected to which elements of the program when reading an algorithm?

\section{METHODOLOGY}

The data analyzed for this paper was obtained as part of a larger, eye-tracking-based experiment. In this experiment, a group of advanced students were tasked with reading and comprehending both programs, i. e., process descriptions, and algorithms, i. e., process 
descriptions together with a proof. The eye-tracking setup and the analysis of the quantitative eye-tracking data obtained is described in a companion paper [19]. Here, we report on the analysis of the qualitative data obtained from retrospective interviews [12] regarding the comprehension process for algorithms.

\subsection{Participants}

Algorithms in the sense of our definition are usually covered in more advanced classes because comprehending them requires knowledge in (1) programming and (2) discrete mathematics. We thus recruited advanced students from the authors' institution that were recommended by instructors to ensure they fit these inclusion criteria Participation in this study was compensated for according to the institutions' standards for trial compensation.

A total of ten students participated in the retrospective interviews regarding the algorithms. All participants were either graduate students or had started working in industry recently. The age range was $22-29$ years. Three participants identified as female; this ratio is representative for the underlying student population.

\subsection{Study Design}

Each session with a participant consisted of three phases. In the first phase, the participant was confronted with different programs. In the second phase, the participant was confronted with two structurally different algorithms. The order in which these algorithms were shown was chosen randomly per participant. During both phases, all eye-movements were tracked and recorded. In the third phase, the participant was confronted with a recording of their eye-movement in a retrospective interview. As only the last two phases are relevant for this paper, we do not discuss the first phase.

The algorithms (FenceLeveling and Plateau) presented in the second phase were selected because they are of similar complexity but exhibit different structural features; these are discussed in detail in Section 3.4. Each algorithm consisted of three components to match the definition we presented in the introduction: (1) a description of a problem domain together with a precise description of what problem the algorithm solves, (2) Java program code that addresses this problem, and (3) a proof why this program code solves the problem. All three components were shown side-by-side on the eye-tracking system's screen throughout each task. No auxiliary means such as pen and paper were allowed to avoid drawing the participants' attention away from the screen.

To allow for assessing whether the participants actually comprehended the algorithms, we deliberately included in the program code of each algorithm one mistake that could easily be overlooked: an off-by-one index error in FENCELEVELING and an erroneous use of a variable in Plateau. We then instructed each participant to first read the problem statement, then the program code and finally the proof in order to comprehend the algorithm. We informed them that one section of the proof would corresponded to a line in the program code containing an error and that they would be asked to identify this line. If they felt they had comprehended the algorithm, they should hit a button which caused that section of the proof to be highlighted. Their task then would be to identify the corresponding line in the program code and to suggest how the flaw might be fixed. All participants were able to identify the flawed line in the program code and attempted-based on the proof-to fix the error.

Based on the recordings of the eye-tracker, all participants' gazes frequently switched between the program code and the proofindicating that their attention switched as well [18]. After they finished working on the algorithm, we presented them with a visualization of their eye-movement during the previous tasks. We conducted retrospective interviews in which we asked to summarize the algorithms and to comment on the recorded eye-movements.

\subsection{Analysis}

The retrospective interviews were recorded with the participants' consent and transcribed in full. Motivated by the frequent eyemovements between program code and proof, we analyzed these transcripts to explore connections between program code and proof.

One common qualitative analysis method used in exploratory studies is inductive content analysis [25]-see, e. g., [21, 34]. We chose to use inductive-as opposed to deductive-content analysis to support the exploratory process instead of limiting the analysis and creating biases, e. g., by building categories only on theories on program or proof comprehension. It is known that in inductive analysis processes "related literature can enhance sensitivity to subtle nuances in data" [6, p. 50]. We thus made sure to consider related literature on proof and program comprehension throughout the analysis. Similarities and differences are discussed in Section 4.

Since each interview addressed two algorithms, we split each interview into two parts. For ease of exposition, we refer to each part as an "interview", i. e., since two algorithms were shown to ten participants each, we ended up with 20 interviews (see "recording units" [25, p. 51]). The first author read each interview line-by-line and marked each sentence describing a relationship between the program code and either the proof or the task (since the proof used terminology from the task, it was unclear at times whether participants referred to the proof or the task). These sentences formed the coding and context units in the sense of Mayring [25, p. 51]. Keeping in mind both the instructional use-case and the use-case of pinpointing errors, we did not distinguish between (factually or logically) correct and incorrect utterances. Based on these coding units, the first author developed two category systems following Mayring's suggestions [25]. The first system described which unit of the program code was connected to the proof or the task (see Table 1). The second system described which unit of the proof or the task was connected to the program code (see Table 2).

The overall coding procedure implemented suggestions of Campbell et al. [4]: The first author marked sentences as described above, developed, and applied the category systems. Then, the second author independently applied the category system to two randomly chosen interviews for each algorithm, i. e., for $20 \%$ of the data. The results were compared, ambiguities discussed, and the category systems refined. After all issues were resolved, the whole material was (re-)coded independently by both authors and the inter-coder reliability was computed over all documents.

As noted above, we were interested in where and how connections between the program code and the proof were made. During the coding process, we made sure to avoid biases from participants repeatedly addressing the same aspect. For this, we recorded for 
each interview whether a connection between two aspects was made, but not how often this occurred during a single interview. To support credibility of the results, we also made sure to not let a single participant influence the kind of connections being reported. We achieved this by reporting a connection between two aspects only if it was coded in at least three interviews, i. e., for at least two participants. The results of the coding are discussed in Section 4.

\subsection{Material}

Because we were interested in understanding the connection between program code and proof, the algorithms used in our study had to consist of a process description along with a proof that was not trivial for the participants. At the same time, the structure of the program code and the proof should be complex, yet different enough to allow for the detection of connections on different levels of abstraction. Based on these inclusion criteria, we chose the FenceLeveling algorithm discussed by Ginat in his "Colorful Challenges" column in the ACM Inroads magazine $[13,14]$ and the Plateau algorithm used by Ginat and Blau in their study on levels of abstraction [15]. In the original exposition, the proof for each algorithm's correctness starts with a short lemma describing a crucial property upon which the actual proof is based. As we were interested in exploring connections between the proof and the program code and to reduced the load for the participants, we decided to not include the proof of such a lemma (which did not relate to the program code) in our material. Instead, we just stated the respective lemma and assured the participants that they could take it's correctness for granted when attempting to comprehend the actual proof. The actual proof reflected the order of the program code describing the process: In the case of Plateau, the overarching structure is a case distinction, whereas in the case of FENCELEVeling, the structure is sequential and more compact.

Plateau. The problem statement, proof, and program code of this algorithm are based on Ginat and Blau's paper [15]:

Consider a sequence of $N$ people standing in a row. The body height of each person is represented as a natural number in a fictitious unit. This is represented as a sequence $S=s_{1}, \ldots, s_{n}$, where each member is a natural number, e. g., the sequence $5,5,6,7,6,6,9,8$. Consecutive values, in which the largest and smallest value differ by no more than one, are called a group. In the example above a group would be $6,7,6,6$, but 5, 5, 6, 7 would be no group, because 5 and 7 differ by two. The goal is to find the largest group.

As noted above, we assured the participants that the following crucial property (originally proven in [15]) was correct and that they should feel free to refer to it when comprehending the proof:

If one considers an arbitrary person $s_{i}$, this person could belong to no more than two groups of maximum size: For one group, $s_{i}$ would be the largest person, for the other group it would be the smallest.

Based on this property, the proof continued as follows:
The algorithm begins by considering the first person. Both possible groups this person may belong to have now at least a length of one. Accordingly, the algorithms stores the length of the longest group it had currently considered. After that the algorithm iterates over all persons. Whenever the algorithm considers the person $s_{i+1}$, four possible cases have to be considered how $s_{i+1}$ affects the previous groups, of which the predecessor $s_{i}$ was a member of.

(1) $s_{i+1}$ and $s_{i}$ differ by more than 1 : In this case, $s_{i+1}$ cannot extend a group where $s_{i}$ belonged to, because $s_{i+1}$ is at least by 2 larger than $s_{i}$.

(2) $s_{i+1}$ and $s_{i}$ are equal: In this case $s_{i+1}$ extends both groups $s_{i}$ belonged to.

(3) $s_{i+1}$ is by 1 smaller than $s_{i}$ : In this case, the group in which $s_{i}$ was the minimum is extended as the group in which $s_{i+1}$ is the maximum. Additionally, the group, in which $s_{i}$ was the maximum, ends, because $s_{i+1}$ is 2 less than the maximum. Instead, a new group begins, in which $s_{i+1}$ is the minimum.

(4) $s_{i+1}$ is by 1 larger than $s_{i}$ : Analogously to the third case, the group in which $s_{i}$ was the maximum is extended as the group in which $s_{i+1}$ is the minimum and a new group begins, in which $s_{i+1}$ is the maximum, whereas the group, in which $s_{i}$ was the minimum, ends.

The algorithm considers iteratively all consecutive persons and stores the length of the respective groups. Additionally, it updates always the length of the longest group it has currently seen and returns this value.

The Java program code followed the order of the proof and consists of four cases corresponding to the four cases of the proof. The program code computes the longest subsequence of consecutive elements in the input that differ by no more than one. It iterates over the input and considers in four if-statements the difference between the element at the current position and the element seen in the previous iteration. Leveraging that no element can be part of more than two subsequences at any given time, the program code then only needs to distinguish between four different cases (difference is zero, plus one, minus one, or more than plus/minus one) to update the (length of) the longest candidate subsequence seen so far. After having iterated over the input, the program code returns the length of the longest candidate subsequence. A variable storing the maximum sequence is updated in each case.

Fence Leveling. The problem statement, proof, and program code of this algorithm are based on Ginat's papers [13, 14]:

Consider a sequence of $N$ consecutive columns. Each column consists of multiple tiles. In total there are $N \times H$ tiles. This is represented as the sequence $S=s_{1}, \ldots, s_{n}$, where each member is a natural number, e. g., the sequence $5,6,7,10$, with $H=7$. The goal is to compute the smallest number of moves of tiles, so that all tiles are of height $H$. In each move one could move an arbitrary number of tiles from one column to an adjacent one. In the example above a valid move is to move 3 tiles from the last column to the second last column. This would lead to the configuration 5, 6,10,7. 
Again, the proof began by stating a crucial property:

The first subsequence from the beginning to an index $k$, where $\sum_{i=1}^{k} s_{i}=k \cdot H$ holds, could be transformed in exactly $k-1$ moves and this number is the minimal number of moves.

As in the case of the proof of Plateau, participants were assured that this property was correct and that they should feel free to refer to it when comprehending the proof. Based on this property, the proof continued as follows:

The algorithm computes for each column the number of tiles to its left. In order to do this, the sum of all elements from the beginning to the current index is computed. Using this sum, the first index $k_{0}$ is found, so that from the beginning to $k_{0}$ are exactly as many tiles, so that the tiles can be moved so that all columns from the beginning to (including) $k_{0}$ are of height $H$. It is stored, that such a subsequence of columns was found. Now, the algorithm considers the remaining subsequence, starting from $k_{0}$. In order to do that, the index where the next subsequence starts, is updated. This is done correctly, because the algorithms removes the number of tiles left from the left of $k_{0}$ from the original sum, because $\sum_{i=k_{0}}^{t} s_{i}=\sum_{i=1}^{t} s_{i}-$ $\sum_{i=1}^{k_{0}} s_{i}$. Following this procedure, the index $k_{1}$ is found, so that to the left of $k_{1}$ until $k_{0}$ are exactly as many tiles, so that all columns from $k_{0}$ to $k_{1}$ can be transformed to be of height $H$. This procedure is repeated until the end of the sequence is reached. Every time such a subsequence is found, the number of such subsequences is incremented. Because of the assumption, that the total number of tiles is $N \times H$, each newly found subsequence can be transformed as described above. Eventually, $t$ of such subsequences with $S_{k_{i}}$ tiles are found. If $\sum_{i=k_{0}}^{t} S_{k_{t}}=N \cdot H$ it follows, that all columns can be transformed in $N-t$ moves, so that all columns are of height $H$.

The Java program code ${ }^{1}$ reflects the order of the proof: Given an array $A$ of integers and an integer $h$, the algorithm iteratively identifies prefixes of this array such that the sum of the $s_{i}$ values in each prefix equals $h \cdot s_{i}$. It then returns the difference of the length of the array and the number of such prefixes. The program code first uses a for-loop to compute an array storing all prefix-sums. It then iterates over this array and increments a counter for each index $i$ such that $A[i]=i \cdot h$ holds. After the last iteration, the program code returns the difference between the length of the prefix-sum array and the value of this counter.

\section{FINDINGS AND DISCUSSION}

As mentioned above, we coded statements that considered the proof or the domain as well as the program code. Therefore, we were able to assign each statement two codes; one code regarding the program code (Table 1) and one code regarding the proof or the domain (Table 2). This allowed for an category-based analysis of connections between the program code and the proof or the domain.

To increase the credibility we only considered connections between categories that appeared in at lest three documents, i. e.,

\footnotetext{
${ }^{1}$ The program code used in this study can be found at http://go.wwu.de/9h4ps.
}

\begin{tabular}{|c|c|c|}
\hline Name & Description & Anchor Example \\
\hline $\begin{array}{l}\text { Existence of } \\
\text { Variables }\end{array}$ & $\begin{array}{l}\text { Statements about variables in the program code } \\
\text { without explicitly referring to any variable. Most } \\
\text { statements in this category are rather on a meta- } \\
\text { level, referring to general approaches or initial } \\
\text { descriptions of the own comprehension process. }\end{array}$ & $\begin{array}{l}\text { C: I think I tried to match the variables in } \\
\text { the proof with the variables in the code. }\end{array}$ \\
\hline $\begin{array}{l}\text { Paradigm } \\
\text { and Design } \\
\text { Technique }\end{array}$ & $\begin{array}{l}\text { Statements referring to the superficial structure } \\
\text { of the program code, e. g., the number of if-else- } \\
\text { cases, are assigned this category. This category } \\
\text { only applies, if the description is only concerned } \\
\text { with superficial aspects of the program code and } \\
\text { no understanding of the actual program code can } \\
\text { be found in the coded segment. }\end{array}$ & $\begin{array}{l}\text { F: In the beginning I tried, uhm,to under- } \\
\text { stand the proof and especially when the } \\
\text { cases down there came, it was very nice, that } \\
\text { one somehow, that it was very convenient, } \\
\text { that one can compare the, uhm, code, also } \\
\text { how these four cases are solved in the code } \\
\text { and if this really are these four cases. }\end{array}$ \\
\hline $\begin{array}{l}\text { Reference } \\
\text { to a Variable } \\
\text { by Name }\end{array}$ & $\begin{array}{l}\text { Statements that refer to an actual variable in the } \\
\text { program code, without any details how this vari- } \\
\text { able is used or any other information. This cat- } \\
\text { egory is less superficial than the previous cate- } \\
\text { gories, but is still rather superficial, because, if } \\
\text { any details regarding the variable are mentioned, } \\
\text { the category Action on a variable would apply. }\end{array}$ & $\begin{array}{l}\text { A: There I tried to find the correspondence } \\
\text { between the variables } \mathrm{s} 1, \mathrm{~s} 2 \text {, which I didn't } \\
\text { fully understand while reading the code, } \\
\text { what exactly they represent. Because their } \\
\text { names were similar in the proof sketch this } \\
s_{i} \text { and } s_{i+1} \text {, I reckoned that } s_{i} \text { and } s_{i+1} \text { had } \\
\text { something to do with } \mathrm{s} 1, \mathrm{~s} 2 \text {. }\end{array}$ \\
\hline $\begin{array}{l}\text { Action on a } \\
\text { Variable }\end{array}$ & $\begin{array}{l}\text { Statements referring to a variable in the program } \\
\text { code and how it is used, e. g., updating the vari- } \\
\text { able or storing a value in it. Description of vari- } \\
\text { able functions, e. g., a counter-variable, were often } \\
\text { used interchangeably and thus also assigned to } \\
\text { this category. }\end{array}$ & $\begin{array}{l}B:[\ldots] \text { then we look for the next span. And } \\
\text { for this, the variable s, which marks the } \\
\text { beginning of the span, I just can't find a } \\
\text { better word, uhm, is marked, uhm, updated. }\end{array}$ \\
\hline $\begin{array}{l}\text { Control- } \\
\text { Flow }\end{array}$ & $\begin{array}{l}\text { Statements referring to the a single line of pro- } \\
\text { gram code, e. g., a control structure, or even parts } \\
\text { of this line, such as a description of a loop-header } \\
\text { or a if-statement are assigned this category. If } \\
\text { multiple lines of program code are addressed, the } \\
\text { category sumMARY would apply. }\end{array}$ & $\begin{array}{l}F: \text { We always considered the predecessor and } \\
\text { the current one }[\ldots] \text { and that was this com- } \\
\text { parison. }\end{array}$ \\
\hline $\begin{array}{l}\text { Naive } \\
\text { Algorithm }\end{array}$ & $\begin{array}{l}\text { Statements describing a program or program sec- } \\
\text { tions based on ideas of the participants. Some par- } \\
\text { ticipants described a naive algorithm that solves } \\
\text { the problem presented in the material. Meta- } \\
\text { statements mentioning that a participant consid- } \\
\text { ered developing an naive algorithm are also as- } \\
\text { signed this category. }\end{array}$ & $\begin{array}{l}\text { H: So, I understood what this group of per- } \\
\text { sons is [...] One can, uhm, if one thinks } \\
\text { about how to solve the task, then it's some- } \\
\text { thing different to have a complete solution. }\end{array}$ \\
\hline Summary & $\begin{array}{l}\text { Statements summarizing multiple lines of pro- } \\
\text { gram code. This may be a summary of a single } \\
\text { case of the program code or even summaries of } \\
\text { multiple sections. }\end{array}$ & $\begin{array}{l}\text { F: the last ifs are similar, if one person is } \\
\text { larger, but only larger by } 1 \text { or smaller, then, } \\
\text { uhm, we could increase one group. That } \\
\text { means, either, uhm, the group where the cur- } \\
\text { rent person is the largest one or the group } \\
\text { where the current person is the smallest one. }\end{array}$ \\
\hline Parameter & $\begin{array}{l}\text { Statements referring to the programs parame- } \\
\text { ter(s). }\end{array}$ & $\begin{array}{l}\text { B: This was the task where we were given a } \\
\text { column of tiles and in the end we wanted to } \\
\text { have all columns on the same high, where } \\
\text { the height was a parameter or our function. }\end{array}$ \\
\hline $\begin{array}{l}\text { Summary of } \\
\text { the whole } \\
\text { program }\end{array}$ & $\begin{array}{l}\text { Statements summarizing the whole program, but } \\
\text { not describing it on a section-by-section level. }\end{array}$ & $\begin{array}{l}\text { B: The program finds the minimal number } \\
\text { of moves, that are required, if one wants to } \\
\text { move tiles between the columns to reach the } \\
\text { goal. }\end{array}$ \\
\hline
\end{tabular}

Table 1: Categories used to describe aspects of the program code. The last two categories did not occur in at least three interviews and are thus not retained for display in Figure 1.

connections that were established by at least two participants. After completing the inductive category development we arrived at nine categories related to proof and domain and-incidentally-also nine categories related to the program code.

As described in Section 3, all interviews were coded by both authors. The final inter-coder reliability on all documents was $\kappa=$ .756, an indicator of substantial agreement between the coders [22]. The connections between categories both authors arrived at was therefore also very similar; they agreed on 12 out of 14 connections. For the analysis, both authors agreed to proceed with the coding of the first author, who had developed the coding scheme. All resulting kinds of connections are presented in Figure 1. Most connections appeared in three or four documents. The connection between "Comments on a Variable" and "Reference to a Variable by Name" (6 times), between "Generic Comment" and "Paradigm and Design Technique" (7 times) and between "Artificial Construct/ Entity" and "Action on a Variable" (15 times) appeared more frequently. 


\begin{tabular}{|c|c|c|}
\hline Name & Description & Anchor Example \\
\hline $\begin{array}{l}\text { Generic } \\
\text { Comment }\end{array}$ & $\begin{array}{l}\text { Statements regarding the proof's superfi- } \\
\text { cial structure or commenting on the ex- } \\
\text { istence of variable (see also existence of } \\
\text { variables in the code). }\end{array}$ & $\begin{array}{l}\text { A: I realized there is a huge case distinction with } \\
\text { four cases in the proof sketch. And these four cases } \\
\text { are also prominent in the program, in the loop. }\end{array}$ \\
\hline $\begin{array}{l}\text { Comments } \\
\text { on Variable } \\
\text { Names }\end{array}$ & $\begin{array}{l}\text { Statements referring to variables in the } \\
\text { proof by name or comments on the choice } \\
\text { of names. }\end{array}$ & $\begin{array}{l}\text { D: This is exactly the match between what is } \mathrm{s} 1 \text { and } \\
\text { what is } \mathrm{s} 2, \text { uhm, so one has to translate between } \\
\text { the variables how they are named in the proof and } \\
\text { this is why I look into the left part [, the code]. }\end{array}$ \\
\hline $\begin{array}{l}\text { Trigger } \\
\text { Wording }\end{array}$ & $\begin{array}{l}\text { Statements stressing a word or a phrase } \\
\text { from the proof, e. g., Counting or Updating. }\end{array}$ & $\begin{array}{l}\text { C: [...] 'cause the code is rather short and, uhm, } \\
\text { the updating of the index, I mean, there's like only } \\
\text { on location where such an updating of the index } \\
\text { happens. That } \mathrm{c} \text { is a counter and before that we } \\
\text { only compute partial sums and write 'em back. }\end{array}$ \\
\hline $\begin{array}{l}\text { Artificial } \\
\text { Construct/ } \\
\text { Entity }\end{array}$ & $\begin{array}{l}\text { Statements referring to terms used to de- } \\
\text { scribe the problem. Originally, we distin- } \\
\text { guished rather artificial terms to argue } \\
\text { about the problem statement (such as sub- } \\
\text { sequences) and rather concrete terms (e. g., } \\
\text { plates or persons) situating the problem in } \\
\text { a real world context. }\end{array}$ & $\begin{array}{l}\text { B: This means we actually create a sequence of } \\
\text { columns and eventually it satisfies a condition. And } \\
\text { then we are done and we begin with the next se- } \\
\text { quence of columns by moving this index } \mathrm{s} \text {. }\end{array}$ \\
\hline $\begin{array}{l}\text { Self-Created } \\
\text { Term }\end{array}$ & $\begin{array}{l}\text { Statements referring to terms that were } \\
\text { not provided by the material are assigned } \\
\text { this category. }\end{array}$ & $\begin{array}{l}\text { D: I mean, maybe that's obvious, but one wants } \\
\text { to be sure that } \mathrm{b} \text { is the last number of the second } \\
\text { window. }\end{array}$ \\
\hline $\begin{array}{l}\text { Isolated } \\
\text { Part of the } \\
\text { Proof }\end{array}$ & $\begin{array}{l}\text { Statements summarizing an element of the } \\
\text { proof, e.g. a formula, how a variable in the } \\
\text { proof is modified or what a specific proof } \\
\text { section is about (without understanding } \\
\text { the details of the reasoning). }\end{array}$ & $\begin{array}{l}\text { C: Then I was able to look on a higher level, if I } \\
\text { could recognize any blocks. For example the finding } \\
\text { of such a partial area that sums up, so it yields } k \cdot h \text {. }\end{array}$ \\
\hline $\begin{array}{l}\text { Free } \\
\text { Reasoning }\end{array}$ & $\begin{array}{l}\text { Statements formulating the ideas of the } \\
\text { proof or parts of the proof in own words or } \\
\text { connecting cause and effect of some parts } \\
\text { of the proof. }\end{array}$ & $\begin{array}{l}\text { H: And that was the first block where the two values } \\
\text { differ by two. So that was our termination condition, } \\
\text { that a group ends there, because we had this, that } \\
\text { if the difference was greater by one, we meet our } \\
\text { termination condition and reset } \mathrm{s} 1 \text { and } \mathrm{s} 2 \text {. }\end{array}$ \\
\hline Example & $\begin{array}{l}\text { Statement in which an example is used, } \\
\text { e. g., to simulate the code. }\end{array}$ & $\begin{array}{l}\text { B: Here I looked at the sequence } 5,6,7,10 \text { and then } \\
i \text { thought okay, if } h \text { has now the value } 5 \text { and the } \\
\text { first span would consist only of the first element, } \\
\text { what would be sorted correctly. }\end{array}$ \\
\hline $\begin{array}{l}\text { Prose } \\
\text { Description } \\
\text { of Behavior }\end{array}$ & $\begin{array}{l}\text { Statements regarding a prose description } \\
\text { in the proof. Because no prose description } \\
\text { was included in the proofs, those state- } \\
\text { ments were usually meta-statements about } \\
\text { exceptions. }\end{array}$ & $\begin{array}{l}\text { G: The proof sketch describes how the algorithms } \\
\text { proceeds on an abstract level. The code implements } \\
\text { this then. }\end{array}$ \\
\hline
\end{tabular}

Table 2: Categories used to describe aspects of the proof or task. The last two categories did not occur in at least three interviews and are thus not retained for display in Figure 1.

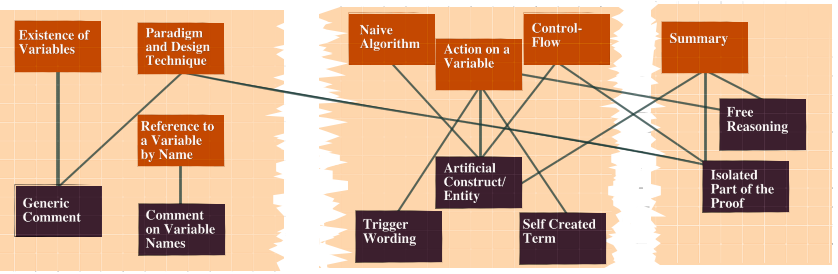

Figure 1: Category-level connections that were established by at least two participants. The categories shown as orange boxes refer to the program code, whereas the categories shown as brown boxes refer to the proof or task.

\subsection{Most connections are on similar levels of abstraction}

Both algorithms were represented used Java program code, a problem description (task), and a proof of correctness. Theories of program and proof comprehension often describe different levels of abstraction. To allow for comparing to these, we structured our category systems according to levels of abstractions as well. For this, we were guided by Biggs's Structure of Observed Learning Outcomes (SOLO) taxonomy and arrived at three levels of abstraction:
An abstraction on the lowest level corresponds to Biggs's prestructural understanding, showing no comprehension at all or comprehension at most "at the individual word level" [1, p. 39], but not going beyond a surface comprehension. "Referring to a Variable by Name" is on this level, as such a superficial utterance with no reference to any broader context does not reveal deeper understanding.

An abstraction on the middle level resembles Biggs's uni- or multistructural comprehension. Statements on Biggs's unistructural level meet only one aspect of the task and describe aspects only in isolation, e. g., understanding a single line of code. Statements on Biggs's multistructural level are described as a "disorganized collection" of unistructural aspects [1, p. 49]. Since our category systems are applied to sentence-level coding units, we consider these two types of statements to be on the same level of abstraction.

An abstraction on the highest level contains categories related to Biggs's relational and extended abstract leveled. Responses on a relational level organize multiple aspects and understanding "their contribution to the topic as a whole" [1, p. 40], e. g., multiple lines of code are summarized. Responses on an extended abstract level go "beyond what has been given" [1, p. 40]. Keeping in mind that we were working with advanced students and Biggs's statement that "today's extended abstract is tomorrow's relational" [1, p. 40] we decided to treat categories on these two SOLO levels to be on the same level of abstraction for our intents and purposes.

Figure 1 shows the connections that were found through our analyses. On the lowest level (left area) we show features of the program code and the proof or task that required only a superficial understanding of the algorithm. Statements connecting elements on this level of abstraction usually described general strategies, such as identifying matching variables in proof and program code or recognizing similarities in the structure of the material:

A: I realized there is a huge case distinction with four cases in the proof sketch. And these four cases are also prominent in the program, in the loop.

While, in general, such prestructural [1] connections "might miss the point" of the algorithm, they can nonetheless be relevant for the overall comprehension process: Narayanan and Hegarty's model of multimedia comprehension explicitly incorporates such parsing of small units of the material [28, pp. 282-283]. In an early stage they then describe "coreference resolution, i. e., making referential links between elements of the different representations that refer to the same entity" [28, p. 284]. This comprehension level is considered a base for connections between different representations, e. g., a figure illustrating parts of some object and a textual description of its function. We note that models of proof comprehension [26, 40] and program comprehension [33], both of which do not deal with relating different constructs, do not consider such a prestructural level, so this level appears to be needed for coreferencing only.

To support comprehension of this level, Narayanan and Hegarty suggest that the material "should explicitly support accurate and early parsing" [28, p. 283] and that the material should make connection explicit to support coreference resolution [28, p. 284]. As we can see in the example above, Participant A utilized that four cases were prominent in both the program code and the proof and connected them. This parallel constructions showcases how such 
connections can be fostered in the domain of algorithms; the corresponding sections refer to one case to be considered each and they stood out in the representation. The program code describes how this case is handled and the proof argues why this is correct. In Section 4.3, we further elaborate on such connections and how these contribute to the overall comprehension process.

Our middle level of abstraction resembles Biggs's unistructural and multistructural level, in which one or multiple aspects are understood in isolation but not connected [1]. Statements on this level address isolated components, e. g., specific wording, certain domain entities, or indicators of understanding isolated lines of the program code, e. g., modification of variables. Most statements on this level described lines of program code in terms of the domain:

B: [...] then we have this mean comparison with the multiplication of the indices, which I didn't understand until the end. But this product is exactly the height of the the column.

Statements on the lowest level of abstraction did not require processing of the material in detail: superficial features could be noted even if the material would be presented in a foreign language. In contrast, statements on the middle level require comprehension of details; the material is processed in detail, but still in isolation.

We relate this level of abstraction to theories from program comprehension and proof comprehension. In, e. g., the block model of program comprehension, understanding single lines of program code in isolation is modeled as the so called atom level [32]. Similarly, the basic facet of Yang and Lin's model of proof comprehension considers the comprehension of proof elements in isolation, e. g., being able to identify angles in a triangle or understand the meaning of congruence in a given triangle. Therefore, the connections observed on our middle level of abstraction connect indicators of low-level program comprehension and low-level proof comprehension.

The proofs of both our algorithms used specific wordings describing the code, e.g., "updated". Even though, only interviews pertaining the FENCELEVELING algorithms showed participants relating the specific wording from the proof to "actions on variables" (4 out of 10 interviews); no such connection was found in the interviews pertaining to PlateAU. A closer look revealed that the main reason given for establishing such a connection was the (short) length of the code and the fact that not too many suitable matches for the trigger wording in the program code existed:

C: [...] 'cause the code is rather short and, uhm, the updating of the index, I mean, there's like only on location where such an updating of the index happens. That $c$ is a counter and before that we only compute partial sums and write 'em back.

This illustrates that the design guidelines suggested by Narayanan and Hegarty [28] are also relevant for higher levels of abstraction. An implication for educators is that the wording used in a proof of algorithms with longer program code may not be a reliable way to foster connections between proof and program code.

Our highest level of abstraction resembles Biggs's relational and extended abstract level, in which multiple aspects are connected to each other or the statement goes "beyond existing principles" [1, p. 41]. All categories in this level go beyond understanding concepts in isolation; we model, e. g., summaries of program code sections, including the connection of multiple lines of program code or free explanations of the proof's reasoning. Most connections on this level were summaries of program code sections in terms of the domain or reasons why the program code worked correctly or not:

F: the last ifs are similar, if one person is larger, but only larger by 1 or smaller, then, uhm, we could increase one group. That means, either, uhm, the group where the current person is the largest one or the group where the current person is the smallest one.

The perspective that a connection of isolated parts describes an advanced state of comprehension is also reflected in related theories: In Yang and Lin's model of proof comprehension, the comprehension of the role of statements in a chain of reasoning is considered an higher level of comprehension: they describe connecting premise and conclusion in a given proof as a higher level of comprehension than just comprehending these in isolation [40, p. 66]. In program comprehension, a summary of multiple lines of program code as a block or the comprehension of the relationship of these blocks marks the next step of comprehension [24, 32]. In comprehension of physical systems, Narayanan and Hegarty described similar phenomenona and explained that "components of a machine are often made up of subcomponents" [28, p. 283].

Considering our middle level of abstraction, we found that participants understood aspects in isolation regarding the program code as well as regarding the proof and the domain. Considering our higher level of abstraction, we observed that participants connected these aspects to reach other aspects in terms of the program code or the proof and domain. This progression is one dimension in which comprehension can be described [26, 28, 33, 40]. However, we also found a crosscutting dimension: participants connected aspects referring to the program code and aspects referring to the proof or the domain: As Figure 1 illustrates, most connections remain within an area, i. e., mostly link categories on a similar level of abstraction. In this way, algorithm comprehension describes an additional dimension of comprehension that goes beyond existing theories on proof or program comprehension; we explore this further below.

\subsection{The task description may have a higher impact on the domain model than the rest of the problem statement or the proof}

By design, our material included for each algorithm a precise description of a problem the algorithm addressed. The problem and the proof used terminology from the problem domain, e. g., people of different sizes, or introduced formalized, artificial constructs such as subsequences, to reason about the problem and the process solving it. The participants used these terms to describe the program code line by line or to even summarize entire sections:

D: [...] it sums all up, the number of tiles, so it's the sum of these.

Both the proofs and problem statements of the two algorithms contained artificial constructs, e. g., "groups" or "subsequence", and terminology from the problem domain, e. g., "person" or "move".

The task Plateau addressed was worded as "finding the largest group", i. e., used an artificial construct. The proof also explicitly 
referred to two groups: the current candidates for the largest group. In contrast, the task FenceLeveling addressed was worded as "finding the smallest number of moves", i. e., used terminology from the domain. However, the proof referred to subsequences and the number of tiles in such a subsequence, i. e., an artificial construct. The number of moves, i. e., terminology from the domain, was only referred to in the last section of the proof.

Quite unexpectedly, we found a difference between the algorithms regarding the use of terminology from the problem domain or artificial constructs to describe the program code: Whereas 9 out of 10 participants used the name of an artificial construct (in this case: "group") to describe an action on a variable in Plateau, only 3 out of (the same) 10 participants did so for FenceLeveling, even though the program code considered an artificial construct as well (subsequences). Moreover, except for one occurrence all artificial constructs participants used to describe the code of FenceLEveling were not based on the material. Instead, the participants created their own terms to describe the code (category Self-Created Term):

B: $[\ldots]$ then we look for the next span. And for this, the variable s, which marks the beginning of the span, I just can't find a better word, uhm, is marked, uhm, updated.

For FenceLeveling, all references made to the "number of moves" (a term from the domain) the algorithm computes, were found to be inconsistent with the material. These references described each increase of a counter $c$ as "counting the moves"; this counter, however, keeps track of the number of subsequences-an artificial construct-and is used only later to compute the number of moves:

$\mathrm{E}:[\ldots]$ I think the counter, that we increase, this $\mathrm{c}$, that has to be the number of moves.

Only one participant connected the subsequences to the program code at all. In contrast, four participants incorrectly related the increment of $c$ in the program code to the number of moves, even though they commented on sections of the proof before and after which were unrelated to the number of moves. The other five participants did not connect any artificial construct or domain term to the program code. On the other hand, all ten participants (correctly) related the two groups-an artificial construct-defined in the proof to variables in the program code.

Narayanan and Hegarty suggest, that "referential links between elements of the different representations that refer to the same entity" should be supported by design choices [28, p. 283]. A task formulation such as in PLATEAU which was taken up in the proof seems to leads to more connections between the domain and the program code and therefore supports establishing connections. Conversely, a task formulation such as the one in FENCELEVELING which is hardly ever taken up in the proof seems to lead to fewer and at times even incorrect domain/program code connections.

We hypothesize that the task formulation has a significant influence on building a domain model into which the algorithm is embedded. The program code can then be connected to entities from this domain. If the proof argues about the same entities, referential links are easier to establish and more likely to be correct.

\subsection{Superficial features may help navigating the material}

To assess whether our participants had successfully comprehended the algorithm, we had introduced an off-by one error in FENCELEVELING and an erroneous use of a variable in PLATEAU, both minor enough that no participant spotted them during the first comprehension pass. After the participants indicated that they felt they had comprehended the algorithm, a section of the proof which argued about the erroneous line of code in the program was highlighted. The participants were asked to identify the erroneous line of code and to suggest a fix for that error. All participants successfully identified the exact location of the flaw after the proof section was highlighted. To understand the highlighted section, they had to understand the chain of reasoning in the proof. To locate (and to fix) the error, they had to understand the program code on a detailed level (Action on a Variable) and connect this understanding to the proof. Together this would imply that locating and fixing the error would indicate that such a connected understanding existed and could be leveraged. During the interviews, three participants explicitly mentioned this kind of connection. Two of these connected the reasoning to a summary of the flawed code section:

F: There at s1++ was the error, because the person, if the group is larger by one, uhm, when the person for the group is bigger by one, uhm, then the other group where this person was the largest one can't be extended, because then within this group, uhm, there would be a difference of two.

The other participant connected incrementing a variable with the reasoning, but was uncertain if he had chosen the correct variable.

C: We have s1 and s2 and we increment both by one. That means that one of these must be wrong, because we can only increment one group but not the other. But I don't know if I was irritated by the proof or if I understood that right, but the groups are restricted by the maximum and the minimum and they switch their roles. And that's why I wasn't sure which of these two variables had which role, because they switches the whole time.

This insecurity might have resulted from not understanding the line of code, especially the role of the variables, in its context. This illustrates the importance of connection on both high and low levels of abstraction: Low levels are needed to identify isolated lines of code, e. g., to locate an error, while high levels are needed to put them into context, e. g., for fixing an error.

While the previous observation illustrated that comprehension on higher levels of abstraction supports fixing errors, the following observation illustrates, how lower levels of abstraction may support the development of higher levels of abstraction: The proofs of both algorithms reflected the order of the program code. However, Plateau also consisted of four cases that were distinguished prominently in proof and program code. In addition, variable names in the proof and the program code for PlATEAU were very similar, e. g. $s_{i}$ (proof) and $s 1$ (program code). In FenceLeveling, on the other hand, variable names were not always that similar: while the height $H$ (proof) was modeled by a variable $h$ in the program code, the 
index $k_{i}$ (in the proof) indicating the end of the last subsequence was modeled by a variable $s$ in the program code.

Three of the four participants commenting on the superficial structure of the program code in connection to the proof stated how it was used to pinpoint certain details from the proof:

\section{G: I knew that in the end I would be given a location in the proof where something would be wrong and that I would just have to fix the location in the code and the correlation is quite obvious even without looking at how, for exam- ple, the third case is implemented.}

Comparing the two algorithms revealed two main differences regarding superficial features. The first difference was that the superficial structure was referred to more often for PLATEAU (8 out of 10 interviews) than for FENCELEVELING (3 out of 10 interviews). In particular, all of these participant commented on the very prominent case distinction in Plateau. Even though the order of the proof of FENCELEveling reflected the order of the program code as well, only one participant noticed and utilized this: When asked how he had located the flaw in the program code he said that he had leveraged that "the proof had the same order as the program code".

The second difference was that variables in the program code were referred to by name in 7 out of 10 interviews in PLATEAU, for example to match these to variables in the proof:

A: There I tried to find the correspondence between the variables $\mathrm{s} 1, \mathrm{~s} 2$, which I didn't fully understand while reading the code, what exactly they represent. Because their names were similar in the proof sketch this $s_{i}$ and $s_{i+1}$, I reckoned that $s_{i}$ and $s_{i+1}$ had something to do with $\mathrm{s} 1$ and $\mathrm{s} 2$.

In contrast, the name of variables were commented on in only 3 out of 10 interviews for FenceLeveling. Participant D even noticed that the lack of similarity hindered him from connecting variables in the proof and the program code for FenceLeveling:

D: If one writes down proofs, that should of course be named

$k_{0}$, that would have been easier. [...] if one makes it consistent, it will be much easier.

These differences show that the similarities in the superficial structure and naming schemes for variables in the proof and the program code of PlateAu may have caused more connections being established on the lowest level of abstraction. Looking at the absolute number of connections being made, i. e., not just checking whether at all such a connection was made in one interview, we found 61 connections of the lowest level of abstraction in for Plateau and 41 such connections for FenceLeveling. This tendency remained on the two higher levels as well: 36 connections were mentioned for PlATEAU and 28 connections were mentioned for FenceLeveling. Most prominently, we found more than twice as many connections involving the Free Reasoning category for Plateau (8 connections) than for Fenceleveling ( 3 connections).

Relating this to theories on program comprehension, we note that Schulte et al. [33] report that some theories [11] emphasize that navigation within different abstractions of a program is rooted in the recognition of basic patterns in some models. In line with this, we found that such basic patterns may also be beneficial for navigating the program code and the proof, e. g., for pinpointing sections from the proof in the code. The algorithm with more prominent similarities-the overall structure and variable names-did not only lead to an increased number of superficial connections but also a higher number of connections on higher levels of abstraction.

\section{CONCLUSION AND LIMITATIONS}

We studied the connection between the program code and the proof of an algorithm through an inductive qualitative content analysis of data obtained from retrospective interviews. The recall stimulus for these interviews were traces of the participants' gazes when attempting to comprehend an algorithm and to fix a mismatch between the proof and the program code; these traces were obtained through a state-of-the art eye-tracking system.

We found that participants described both the program code and the proof of the algorithm on varying levels of abstraction. As part of our analysis, we grouped the categories assigned to these descriptions regarding their level of abstraction. These levels of abstractions resemble (groups of) levels of the SOLO taxonomy [1].

While the basic hierarchy of abstractions unsurprisingly resembles hierarchies known from program comprehension and proof comprehension, the connections between elements of the program code and the proof formed a new dimension; this creates a model of algorithm comprehension which cannot be reduced to either program comprehension or proof comprehension alone. The connections between the program code and the proof we found were mostly between elements on similar levels of abstraction. Some actions, however, that required a deeper understanding, e. g., to find and fix an error, appeared to require participants to connect multiple levels of abstraction. We also saw that connections on lower levels of abstraction supported the development of connections on higher levels of abstraction. Finally, we found evidence that the domain the mental model of the algorithm is situated in can be influenced strongly by how the task description is contextualized and worded.

Limitations, Validity, and Meaningfulness. The exploratory character of this study and the limited number of hand-selected participants drawn from a single institution obviously require follow-up research to revalidate and possibly further refine our findings. In addition, both the categories derived as well as their connection should be explored further. For example, summaries of code sections resemble strongly concepts from research on subgoal labels (see e.g. [27]), so exploring these in connection with the proof seems promising. We addressed other possible limitations of our study in different ways: To assure that an actual comprehension process had taken place, i. e., that participants had built a model of the algorithm they could resort to, we had instructed them to read the algorithm keeping in mind that they would be asked to fix a minor mismatch between the program code and the proof; a task they could not attempt unless they had understood both the program code and the proof before. As discussed above, all participants succeeded at this; the fact that no participant had spotted the mismatch during the initial reading pass indicates that this mismatch was not too obvious. Addressing the small number of participants, in particular, to avoid a single participant biasing the derived model of connection, made sure to only model connections between categories that were 
made by at least two participants. We also only checked whether a participant did connect two categories, not how often.

We attempted to establish at least a basic level construct validity by following Mayring's suggestion to compare our findings to related theories [25, p. 111], in this case, program comprehension and proof comprehension. This comparison also serves Tracy's recommendations to "attentively interconnect literature reviewed with research foci, methods and findings" to achieve meaningfulness, i. e., coherence with "espoused theories and paradigms" [37, p. 848]. These comparisons resulted in similarities supporting the construct validity of our findings and differences illustrating how algorithm comprehension cannot be subsumed under either proof comprehension or program comprehension alone.

Implications. We found that structural elements, such as case distinctions or variable names, should be similar in program code and the proof in order to support coreference resolution, i. e., "making referential links between elements of the different representations that refer to the same entity" [28, p. 284]. At the same time, specific (technical) wording, such as "updating" or "storing" appeared to be only utilized by participants if the code was sufficiently short and no ambiguities existed. To support early parsing and navigation in the material, it thus may be helpful if these features would be prominent while superficially scanning the material. An added benefit of pointing out connections on this superficial (and thus seemingly uninteresting) level would be that these seem to support connections on more advanced levels of abstraction as well.

Finally, the description of the task the algorithm addresses may influence a model of the domain the algorithm is situated in. This finding extends Ginat and Blau's [15] observation that algorithms utilize aspects of the domain to achieve certain properties from the scope of properties to the comprehension process.

We thus conclude that the both the structure and the wording of the task description, the program code, and the proof should be as aligned as possible to increase coherency and support coreference resolution, which in turn support algorithm comprehension.

\section{REFERENCES}

[1] John B Biggs. 2011. Teaching for quality at university: What the student does. McGraw-hill education (UK), London, UK.

[2] Ruven Brooks. 1983. Towards a theory of the comprehension of computer programs. Intl. J. Man-Machine Studies 18, 6 (1983), 543-554.

[3] Jean-Marie Burkhardt, Françoise Détienne, and Susan Wiedenbeck. 2002. ObjectOriented Program Comprehension: Effect of Expertise, Task and Phase. Empirical Software Engineering 7, 2 (2002), 115-156.

[4] John L Campbell, Charles Quincy, Jordan Osserman, and Ove K Pedersen. 2013. Coding in-depth semistructured interviews: Problems of unitization and intercoder reliability and agreement. Sociol. Methods \& Research 42, 3 (2013), 294-320.

[5] Tao Chen and Tarek Sobh. 2001. A tool for data structure visualization and userdefined algorithm animation. In Proc. 31st Frontiers in Education Conf. TID-2.

[6] Juliet Corbin and Anselm L. Strauss. 2014. Basics of Qualitative Research. SAGE Publishing, Thousand Oaks, CA.

[7] Martha E. Crosby and Jan Stelovsky. 1990. How do we read algorithms? A case study. Computer 23, 1 (1990), 25-35.

[8] Sylvia da Rosa. 2010. The construction of the concept of binary search algorithm. In Proceedings of the 22th Annual Workshop of the Psychology of Programming Interest Group. 100-111.

[9] Sylvia da Rosa. 2015. The construction of knowledge of basic algorithms and data structures by novice learners. In Proceedings of the 26th Annual Workshop of the Psychology of Programming Interest Group. 37-48.

[10] Karl M. Fant. 1993. A Critical Review of the Notion of Algorithm in Computer Science. In Proc. 1993 ACM Conf. Computer Science (CSC '93). 1-6.

[11] Vikki Fix, Susan Wiedenbeck, and Jean Scholtz. 1993. Mental Representations of Programs by Novices and Experts. In Proc. INTERACT '93 and CHI '93 Conf.
Human Factors in Computing Systems (CHI '93). 74-79.

[12] Kerstin Gidlöf, Nils Holmberg, and Helena Sandberg. 2012. The use of eyetracking and retrospective interviews to study teenagers' exposure to online advertising. Visual Communication 11, 3 (2012), 329-345.

[13] David Ginat. 2019. COLORFUL CHALLENGES: Fence leveling. Inroads 10, 1 (2019), 28-29.

[14] David Ginat. 2019. COLORFUL CHALLENGES: Letter game. Inroads 10, 3 (2019), 19-20.

[15] David Ginat and Yoav Blau. 2017. Multiple Levels of Abstraction in Algorithmic Problem Solving. In Proc. 2017 ACM SIGCSE Techn. Symp. Computer Science Education. 237-242.

[16] Scott Grissom, Myles F. McNally, and Tom Naps. 2003. Algorithm Visualization in CS Education: Comparing Levels of Student Engagement. In Proc. 2003 ACM Symp. Software Visualization (SoftVis '03). 87-94.

[17] Bruria Haberman, Haim Averbuch, and David Ginat. 2005. Is it really an algorithm: the need for explicit discourse. In Proc. 10th SIGCSE Conf. Innovation and Technology in Computer Science Education. 74-78.

[18] Marcel Adam Just and Patricia A. Carpenter. 1980. A Theory of Reading: From Eye Fixations to Comprehension. Psychological Review 87, 4 (July 1980), 329-354.

[19] Philipp Kather, Rordrigo Duran, and Jan Vahrenhold. 2021. Through (Tracking) Their Eyes: Abstraction and Complexity in Program Comprehension. ACM Transactions on Computing Education. In Press.

[20] Philipp Kather and Jan Vahrenhold. 2021. Is Algorithm Comprehension Different from Program Comprehension?. In 2021 IEEE/ACM 29th International Conference on Program Comprehension (ICPC). IEEE, 455-466.

[21] Riko Kelter, Matthias Kramer, and Torsten Brinda. 2018. Statistical FrequencyAnalysis of Misconceptions In Object-Oriented-Programming: Regularized PCR Models for Frequency Analysis across OOP Concepts and Related Factors. In Proc. 18th Koli Calling Intl. Conf. Computing Education Research. Article 6, 10 pages.

[22] J. Richard Landis and Gary G. Koch. 1977. The Measurement of Observer Agreement for Categorical Data. Biometrics 33, 1 (1977), 159-174.

[23] Stanley Letovsky. 1987. Cognitive processes in program comprehension. fournal of Systems and Software 7, 4 (1987), 325-339.

[24] Raymond Lister, Beth Simon, Errol Thompson, Jacqueline L Whalley, and Christine Prasad. 2006. Not seeing the forest for the trees: novice programmers and the SOLO taxonomy. ACM SIGCSE Bulletin 38, 3 (2006), 118-122.

[25] Philipp Mayring. 2014. Qualitative Content Analysis: Theoretical Foundation, Basic Procedures and Software Solution. https://nbn-resolving.org/urn:nbn:de:0168ssoar-395173.

[26] Juan Pablo Mejía-Ramos, Evan Fuller, Keith Weber, Kathryn Rhoads, and Aron Samkoff. 2012. An assessment model for proof comprehension in undergraduate mathematics. Educational Studies in Mathematics 79, 1 (2012), 3-18.

[27] Briana B. Morrison, Lauren E. Margulieux, Barbara Ericson, and Mark Guzdial. 2016. Subgoals Help Students Solve Parsons Problems. In Proc. 47th ACM Techn. Symp. Computing Science Education. 42-47.

[28] Hari Narayanan and Mary Hegarty. 2002. Multimedia design for communication of dynamic information. Intl. 7. Human-Computer Studies 57, 4 (2002), 279-315.

[29] Nancy Pennington. 1987. Comprehension Strategies in Programming. In Empirical Studies of Programmers: Second Workshop. 100-113.

[30] Jacob Perrenet, Jan Friso Groote, and Eric Kaasenbrood. 2005. Exploring students' understanding of the concept of algorithm: levels of abstraction. In Proc. 10th SIGCSE Conf. Innovation and Technology in Computer Science Education. 64-68.

[31] Jean Piaget. 1964. La Prise de Conscience. Presses Universitaires de France, Paris.

[32] Carsten Schulte. 2008. Block Model: An Educational Model of Program Comprehension as a Tool for a Scholarly Approach to Teaching. In Proc. 4th Intl. Workshop Computing Education Research. 149-160.

[33] Carsten Schulte, Tony Clear, Ahmad Taherkhani, Teresa Busjahn, and James H. Paterson. 2010. An Introduction to Program Comprehension for Computer Science Educators. In 2010 ITiCSE Working Group Reports. 65-86.

[34] Sue Sentance, Jane Waite, Steve Hodges, Emily MacLeod, and Lucy Yeomans. 2017. "Creating Cool Stuff": Pupils' Experience of the BBC Micro:Bit. In Proc. 2017 ACM SIGCSE Techn. Symp. Computer Science Education. 531-536.

[35] Anna Sfard and Liora Linchevski. 1994. The gains and the pitfalls of reification The case of algebra. Educational Studies in Mathematics 26, 2 (Mar 1994), 191-228.

[36] Tapani Toivonen and Solomon Oyelere. 2019. BiGO: A Toolset to Support CS Students to Learn to Analyze Time Complexities of Algorithms. In Proc. 19th Koli Calling Intl. Conf. Computing Education Research. Article 3, 6 pages.

[37] Sarah J Tracy. 2010. Qualitative quality: Eight "big-tent" criteria for excellent qualitative research. Qualitative Inquiry 16, 10 (2010), 837-851.

[38] Anneliese Von Mayrhauser and A Marie Vans. 1995. Program comprehension during software maintenance and evolution. Computer 28, 8 (1995), 44-55.

[39] Anneliese Von Mayrhauser and A. Marie Vans. 1996. Identification of dynamic comprehension processes during large scale maintenance. IEEE Trans. Software Engineering 22, 6 (1996), 424-437.

[40] Kai-Lin Yang and Fou-Lai Lin. 2008. A model of reading comprehension of geometry proof. Educational Studies in Mathematics 67, 1 (2008), 59-76. 